Check for updates

Cite this: J. Mater. Chem. A, 2019, 7, 24847

Received 12th June 2019

Accepted 30th September 2019

DOI: $10.1039 / c 9 t a 06290 d$

rsc.li/materials-a

\section{On-the-fly catalytic degradation of organic pollutants using magneto-photoresponsive bacteria-templated microcleaners $\dagger$}

\author{
Fajer Mushtaq, ${ }^{\text {*a }}$ Xiangzhong Chen, (D) *a Silvan Staufert, ${ }^{b}$ Harun Torlakcik, ${ }^{a}$ \\ Xiaopu Wang, (DD a Marcus Hoop, ${ }^{a}$ Ann Gerber, ${ }^{a}$ Xinghao Li, ${ }^{c}$ Jun Cai, (ID ${ }^{c}$ \\ Bradley J. Nelson ${ }^{a}$ and Salvador Panéa
}

\begin{abstract}
Increasing accumulation of highly persistent and non-biodegradable organic pollutants in our fresh water sources imposes a threat to human health. Designing novel catalytic materials that can efficiently harness energy from their surroundings to degrade such problematic pollutants is essential. In this work, we fabricated core-shell microhelical robots composed of iron oxideatitanium dioxide $\left(\mathrm{Fe}_{3} \mathrm{O}_{4}\left(\mathrm{aTiO}_{2}\right)\right.$ for UV-visible light driven degradation of organic pollutants in a cost-effective manner. Bio-templating and sol-gel synthesis were employed for a simplified approach to batch-fabricate magnetic photocatalysts. These hybrid microrobots removed $97 \%$ of RhB dye from contaminated water in 75 minutes using UVvisible light ( $k$-value of $0.047 \mathrm{~min}^{-1}$ ). Furthermore, when photocatalytic degradation was performed under continuous magnetic field driven propulsion, $99 \%$ of RhB dye degraded in 40 minutes with a $k$ value of $0.108 \mathrm{~min}^{-1}$. We also observed a strong correlation between the hybrid microhelices' swimming characteristics and their subsequent photocatalytic degradation efficiency. These results were further corroborated using COMSOL simulations.
\end{abstract}

\section{Introduction}

Global water shortages due to rapidly shrinking clean water reserves, increasing industrial activity, and growing world population, is one of humanity's biggest challenges that requires immediate attention. ${ }^{1}$ Water pollution caused by the discharge of toxic organic pollutants such as, antibiotics, pesticides, herbicides, estrogens, synthetic pigments, pharmaceuticals and diagnostic agents into effluents is further exacerbating the water shortage crisis., ${ }^{2,3}$ Many of these substances are highly bio-active and tend to bio-accumulate over time, even when present at very low concentrations (ng $\mathrm{L}^{-1}$ to $\left.\mu \mathrm{g} \mathrm{L}^{-1}\right){ }^{4}$ This renders these problematic pollutants extremely harmful because of their carcinogenic and mutagenic nature. ${ }^{5-7}$ Being highly persistent and non-biodegradable, most of these organic pollutants easily escape conventional water treatment methods

${ }^{a}$ Multi-Scale Robotics Lab (MSRL), Institute of Robotics and Intelligent Systems (IRIS), ETH Zurich, CH-8092 Zurich, Switzerland.E-mail: fmushtaq@ethz.ch; chenxian@ ethz.ch

${ }^{b}$ Micro and Nanosystems, ETH Zurich, CH-8092 Zurich, Switzerland

${ }^{c}$ School of Mechanical Engineering and Automation, Beihang University, No. 37 Xueyuan Road, Haidian District, Beijing 100191, China

$\dagger$ Electronic supplementary information (ESI) available: SEM characterisation of bio-template coated photocatalysts, XRD data, photocatalytic reusability plot, magnetic characterisation of hybrids using VSM, time-lapse images of hybrids under manipulation and COMSOL simulation results, and videos of magnetic manipulation, and COMSOL results. See DOI: 10.1039/c9ta06290d and are discharged into our surface and groundwater. ${ }^{8,9}$ An advanced oxidation process (AOP) to use highly reactive oxygen species, including hydroxyl and superoxide radicals, is one of the most extensively studied approaches to degrade toxic contaminants into harmless products such as $\mathrm{CO}_{2}$ and $\mathrm{H}_{2} \mathrm{O} .^{\mathbf{1 0 - 1 2}}$ Among the various approaches to initiate AOPs, photocatalysis is one of the most promising, where a semiconductor with a suitable bandgap can efficiently absorb light to photogenerate electron-hole pairs. These electrons and holes can then migrate to the surface of the photocatalyst and initiate a series of oxidative and reductive reactions, resulting in the degradation of organic pollutants. ${ }^{13-15}$ The practical use of these catalysts is limited by their high electron-hole recombination rate, which ultimately lowers their photocatalytic efficiency. ${ }^{16,17}$ To overcome these challenges, wide bandgap semiconductors such as titanium dioxide $\left(\mathrm{TiO}_{2}\right)$ are extensively used. However, having a wide bandgap limits the light absorption of these materials to the UV region. ${ }^{18}$ Hence, various strategies have been investigated including chemically modifying the wide bandgap semiconductors with co-catalysts and doping with non-metals, rareearth, and transition metals. ${ }^{19-21}$ Although such approaches have shown improved photocatalytic efficiency, they rely heavily on rare and expensive catalysts and suffer from complicated fabrication processes. $^{22,23}$ Developing photocatalysts with a core-shell architecture composed of a wide bandgap and a small bandgap semiconductor is yet another approach towards tuning the bandgap of a wide bandgap semiconductor 
in a simplified and cost-effective manner. This approach allows the utilization of a broader solar spectrum and dramatically reduces the electron-hole recombination rate by promoting their separation and, thus, increasing their lifetime..$^{20,24-26}$

In order to develop photocatalysts for practical applications it is important to utilize cost-effective materials that can be easily employed in a simple batch-fabrication process. In addition, it is important to design photocatalysts with a high surface area to enhance the surface-driven catalytic reactions. ${ }^{27,28}$ Efficient photocatalysts should possess additional functionalities that allow their complete removal after water treatment to avoid secondary contamination due to their loss. ${ }^{19,20,29}$ This would also make them reusable for multiple treatments and add to their cost-effectiveness. ${ }^{30}$

In this work, we fabricated our photocatalysts using a biotemplating approach by taking advantage of the helical shape of the microalgae sub-species, Spirulina platensis. A simple batch-fabrication synthesis was developed by dip-coating the bio-template with small bandgap iron oxide $\left(\mathrm{Fe}_{3} \mathrm{O}_{4}\right)$ and wide bandgap titanium dioxide $\left(\mathrm{TiO}_{2}\right)$ in two sequential coating steps. Using this approach, core-shell $\mathrm{Fe}_{3} \mathrm{O}_{4} @ \mathrm{TiO}_{2}$ microhelical structures were developed for photocatalytic water remediation. These hybrid microhelices were used to degrade a model organic pollutant, rhodamine $\mathrm{B}(\mathrm{RhB})$, under UV-visible light and natural sunlight. An $\mathrm{Fe}_{3} \mathrm{O}_{4}$ core provides these microstructures with magnetic properties for actuation in a corkscrew motion in $3 \mathrm{D}$, and further allows for their re-collection. We also investigated the relationship between the hybrid microstructures' swimming behavior and their photocatalytic efficiency. The development of these innovative multicomponent hybrid microstructures provides a cost-effective, simple approach towards batch-fabrication of biocompatible magnetic microrobots for enhanced environmental remediation and future biomedical applications.

\section{Experimental}

\section{Synthesis of $\mathrm{Fe}_{3} \mathrm{O}_{4} \mathrm{NP}$ suspension}

The $\mathrm{Fe}_{3} \mathrm{O}_{4}$ NP suspension was prepared using a co-precipitation method. An aqueous solution of $8 \mathrm{mM} \mathrm{FeCl}_{3}$ and $16 \mathrm{mM} \mathrm{FeCl}_{2}$ was prepared in DI water at $50{ }^{\circ} \mathrm{C}$. A $0.5 \mathrm{M} \mathrm{NaOH}$ solution was added dropwise to this mixture, resulting in the formation of a black suspension. This mixture was sonicated for 60 minutes, centrifuged and dispersed in $50 \mathrm{~mL}$ of DI water five times. Next, $100 \mu \mathrm{L}$ of a $5 \mathrm{M} \mathrm{HCl}$ solution was added to the above $\mathrm{Fe}_{3} \mathrm{O}_{4} \mathrm{NP}$ mixture to bring its $\mathrm{pH}$ to 1.8 , followed by sonication for another 60 minutes.

\section{Deposition of $\mathrm{Fe}_{3} \mathrm{O}_{4}$ NPs on Spirulina platensis bio-templates}

$\mathrm{Fe}_{3} \mathrm{O}_{4}$ NPs were deposited on the biological templates using a dip-coating method under ambient temperature and pressure. Before coating, the bio-templates were sonicated in DI water for 5 minutes to reduce their body length to $3-5$ pitches. Next, $1 \mathrm{~g}$ of sonicated Spirulina was added to $50 \mathrm{~mL}$ of the $\mathrm{Fe}_{3} \mathrm{O}_{4} \mathrm{NP}$ suspension and placed on an orbital shaker (Heidolph Rotamax $120,100 \mathrm{rpm}$ ) for 48 hours to avoid agglomeration of the bio- templates during the coating process. The $\mathrm{Fe}_{3} \mathrm{O}_{4}$ coated biotemplates were then collected using centrifugation and washed several times with DI-water.

\section{Fabrication of $\mathrm{Fe}_{3} \mathrm{O}_{4} @ \mathrm{TiO}_{2}$ bio-templates}

$\mathrm{TiO}_{2}$ was deposited on the $\mathrm{Fe}_{3} \mathrm{O}_{4}$ coated bio-templates by a solgel process. $0.5 \mathrm{~g}$ of $\mathrm{Fe}_{3} \mathrm{O}_{4}$ coated bio-templates were dispersed in a mixed solvent containing $90 \mathrm{~mL}$ of ethanol, $30 \mathrm{~mL}$ of acetonitrile and $0.5 \mathrm{~mL}$ of concentrated ammonia solution. This mixture was placed on an orbital shaker and mixed for 30 minutes. Next, $0.5 \mathrm{~mL}$ of tetrabutyl titanate was added to the above mixture under continuous shaking. After 2 hours, the biotemplates were collected using a magnet and washed several times with ethanol. These core-shell bio-templates were then annealed at $450{ }^{\circ} \mathrm{C}$ in air for 2 hours at a ramp rate of $5{ }^{\circ} \mathrm{C} \min ^{-1}$ to convert the amorphous titanium oxide coating to a crystalline anatase $\mathrm{TiO}_{2}$ phase. During this annealing process, the biological template was removed and the final hybrid core-shell $\mathrm{Fe}_{3} \mathrm{O}_{4} @ \mathrm{TiO}_{2}$ microhelices were formed which were hollow with a rough morphology. In this work, we also fabricated microhelical structures that were composed of only $\mathrm{Fe}_{3} \mathrm{O}_{4}$ or $\mathrm{TiO}_{2}$ by performing the individual coatings as described above, followed by annealing under similar conditions.

\section{Material characterization}

The morphology of the resulting nanotubes was studied by scanning electron microscopy (SEM, Zeiss ULTRA 55, Zeiss, Oberkochen, Germany), transmission electron microscopy (TEM, FEI F30, FEI Co., Hillsboro, OR), and scanning transmission electron microscopy (STEM, FEI F30). The composition and crystallographic structure of the nanotubes were analyzed by energy-dispersive X-ray analysis (EDX) and X-ray diffraction (XRD, Bruker AXS D8 Advance, $\mathrm{Cu} \mathrm{K} \alpha$, (Billerica, MA)). The distribution of elements along the microhelices was studied by EDX mapping using HAADF STEM (FEI Talos F200X). The local crystallographic structure was studied by selected area electron diffraction (SAED). UV-vis diffuse reflectance spectra (DRS) was acquired by a Cary 4000 UV-vis spectrophotometer. $\mathrm{BaSO}_{4}$ was used as the reflectance standard. Magnetic characterisation was performed on a powder of hybrid microhelices using a vibrating. sample magnetometer (EZ9 from Microsense).

\section{Photocatalytic experiments}

Photocatalytic experiments were performed to study the degradation of the model organic pollutant $\mathrm{RhB}$ dye in the presence of our microstructures. An RhB concentration of $4 \mathrm{mg} \mathrm{L}^{-1}$ was chosen to perform the degradation experiments. $20 \mathrm{mg}$ of microstructures $\left(\mathrm{Fe}_{3} \mathrm{O}_{4}, \mathrm{TiO}_{2}\right.$ and core-shell $\mathrm{Fe}_{3} \mathrm{O}_{4}$ @$\mathrm{TiO}_{2}$ microhelices) were dispersed in $50 \mathrm{~mL}$ of $\mathrm{RhB}$ solution and were irradiated with UV-visible light (300 $\mathrm{nm}<\lambda<600 \mathrm{~nm}$ ) using a $300 \mathrm{~W}$ xenon lamp (Asahi Spectra, MAX-303) under mechanical agitation, after the adsorption-desorption equilibrium was reached. A UV-Vis spectrophotometer (Tecan Infinite 200 Pro) was used to obtain the fluorescent spectra of RhB over time by taking aliquots of irradiated RhB solution every 15 minutes for 75 minutes. Experiments were performed under 
direct natural sunlight using a similar concentration of RhB and microhelices, and aliquots of irradiated RhB solution were taken every 10 minutes for 50 minutes.

\section{Manipulation experiments}

Hybrid core-shell $\mathrm{Fe}_{3} \mathrm{O}_{4} @ \mathrm{TiO}_{2}$ microhelices were used for the manipulation experiments using rotating magnetic fields. The magnetic manipulation system consists of eight stationary electromagnets with soft magnetic cores, capable of producing magnetic fields and gradients up to $50 \mathrm{mT}$ and $0.5 \mathrm{~T} \mathrm{~m}^{-1}$ at frequencies up to $100 \mathrm{~Hz}^{31}$ The system was integrated with an inverted fluorescence microscope (Olympus IX 81) and videos of manipulated structures were recorded under rotational magnetic fields at $20 \times$ magnification. The manipulation experiments were conducted using hybrid microstructures in DI water with a few drops of Triton-X surfactant inside custombuilt PDMS containers ( $2 \mathrm{~cm}$ in diameter and $1 \mathrm{~cm}$ in depth). Rotating magnetic fields in the range of $2-10 \mathrm{mT}$ with frequencies between 2-26 $\mathrm{Hz}$ were applied to perform the swimming tests. Five measurements were made to obtain average velocities and the subsequent error bars.

\section{Photocatalytic experiments under magnetic manipulation}

Photocatalytic experiments were performed to study the degradation of the model organic pollutant $\mathrm{RhB}$ dye in the presence of hybrid microhelices under continuous magnetic steering. An RhB concentration of $4 \mathrm{mg} \mathrm{L}^{-1}$ was chosen to perform the degradation experiments. $1.2 \mathrm{mg}$ of microstructures $\left(\mathrm{Fe}_{3} \mathrm{O}_{4}, \mathrm{TiO}_{2}\right.$ and core-shell $\mathrm{Fe}_{3} \mathrm{O}_{4} @ \mathrm{TiO}_{2}$ microhelices) were dispersed in $3 \mathrm{~mL}$ of $\mathrm{RhB}$ solution and were irradiated with UV-visible light (300 $\mathrm{nm}<\lambda<600 \mathrm{~nm}$ ) using a $300 \mathrm{~W}$ xenon lamp (Asahi Spectra, MAX-303), after the adsorption-desorption equilibrium was reached. Rotating magnetic field parameters of $10 \mathrm{mT} 2 \mathrm{~Hz}, 10 \mathrm{mT} 12 \mathrm{~Hz}$ and $10 \mathrm{mT} 22 \mathrm{~Hz}$ were applied to the hybrid microhelices for 40 minutes. A UV-Vis spectrophotometer (Tecan Infinite 200 Pro) was used to obtain the fluorescent spectra of RhB over time by taking aliquots of irradiated RhB solution every $10 \mathrm{~min}$ for $40 \mathrm{~min}$.

\section{COMSOL Multiphysics simulations}

COMSOL Multiphysics was used to understand and illustrate the relationship between the swimming speed of a microswimmer swarm and the corresponding pollutant degradation performance. The swarm of microswimmers is represented by a circle with a diameter similar to that of a swarm, as observed in experiments. In this simplified, time-dependent 2D model, the swarm moved along a circular path within a closed, circular vessel filled with a solution of organic pollutant at a concentration of $4 \mathrm{mg} \mathrm{L}^{-1}$. The swarm movement was achieved by a rotating mesh domain, where the rotation speed was chosen to allow the swarm to move at a speed of $0,19.5$ and $125 \mu \mathrm{m} \mathrm{s}^{-1}$ on its circular path. The creeping fluid flow was achieved by a slipping wall condition on the interface to the swarm. The chemical reaction and transport kinetics are defined in the Chemistry and Transport of Diluted Species COMSOL module. In these modules, a reactive radical species degrades the organic pollutant into an inert carbon species. The reactive radical species was modelled as a species with a constant surface concentration of $0.5 \mathrm{pM}$ within the swarm and a diffusion coefficient of 0 , reflecting the short life time of the species. The organic pollutant species were modelled as rhodamine $\mathrm{B}$ and its diffusion coefficient of $4 \times 10^{-10} \mathrm{~m}^{2} \mathrm{~s}^{-1}$ was implemented in the simulation. These values are derived from existing literature. ${ }^{32}$ The other Multiphysics model and material parameters were obtained from the COMSOL Multiphysics material library. The parameters of the chemical reaction and transport kinetics are not completely representative for the conducted experiments, however they serve as an illustration of the accelerated organic pollutant degradation caused by their increased interaction volume with the microswimmers.

\section{Results and discussion}

There is a growing interest in the scientific community to develop innovative materials by taking inspiration from nature to mimic some of their highly evolved and sophisticated biological functionalities. ${ }^{31,33-36}$ In this direction, bio-templating has emerged as a candidate to replicate the diverse morphological architectures and functionalities of biological entities. $^{34,37,38}$ Spirulina platensis (S. platensis), a microalgae subspecies with a helical shape, was chosen as the bio-template to fabricate magnetic microrobots that are photocatalytic under UV-visible light. ${ }^{39,40}$ The fabrication scheme for the coreshell, $\mathrm{Fe}_{3} \mathrm{O}_{4} @ \mathrm{TiO}_{2}$ hybrid microrobots is presented in Fig. 1a. The $\mathrm{Fe}_{3} \mathrm{O}_{4}$ core provides the microrobots with magnetic and visible-light photocatalytic properties, and the $\mathrm{TiO}_{2}$ shell makes the microhelices active under UV-light. ${ }^{41-43}$ A layer of $\mathrm{Fe}_{3} \mathrm{O}_{4}$ was grown on $S$. platensis bio-templates by immersing the biotemplates into a solution of $\mathrm{Fe}_{3} \mathrm{O}_{4}$ nanoparticles (NPs) at ambient temperature and pressure for 48 hours (Fig. 1a(i)). This was followed by separating the $\mathrm{Fe}_{3} \mathrm{O}_{4}$ coated $S$. platensis templates by centrifugation and several cleaning processes in DI-water. Next, the magnetic micro-helices were coated with an amorphous titanium oxide $\left(\mathrm{TiO}_{x}\right)$ layer using sol-gel synthesis (Fig. 1a(ii)), followed by annealing in air at $450{ }^{\circ} \mathrm{C}$ for 2 hours to convert them to a crystalline $\mathrm{TiO}_{2}$ phase (Fig. $1 \mathrm{a}(\mathrm{iii})$ ). Fig. $1 \mathrm{~b}$ and S1a† show scanning-electron microscope (SEM) images of many overlapping $S$. platensis bio-templates. Magnified images of $S$. platensis bio-templates are provided in Fig. $1 \mathrm{c}$ and $\mathrm{S} 1 \mathrm{~b}-\mathrm{d}, \dagger$ from where we can observe that the bio-template's surface is marked by rough and irregular morphology. After the deposition of $\mathrm{Fe}_{3} \mathrm{O}_{4}$ (Fig. 1d, e and $\mathrm{S} 2 \dagger$ ) and $\mathrm{TiO}_{x}$ (Fig. 1f, $\mathrm{g}$ and S3 $\dagger$ ), the rough surface of $S$. platensis is replaced by a smooth morphology, indicative of a uniform material deposition. During annealing, the biological templates are removed, resulting in $\mathrm{Fe}_{3} \mathrm{O}_{4} @ \mathrm{TiO}_{2}$ microhelices that possess a hollow and rough morphology as shown in Fig. 1h, i and S4. $\dagger$ The hybrid microrobots developed in the study have a body length of 50-80 $\mu \mathrm{m}$ and a diameter of approximately $2.8 \mu \mathrm{m}$.

The crystalline structure of the hybrid $\mathrm{Fe}_{3} \mathrm{O}_{4} @ \mathrm{TiO}_{2}$ microhelices was analyzed using X-ray diffraction (XRD) and transmission electron microscopy (TEM). XRD investigation performed on the hybrid $\mathrm{Fe}_{3} \mathrm{O}_{4} @ \mathrm{TiO}_{2}$ microhelices (Fig. 2a and 

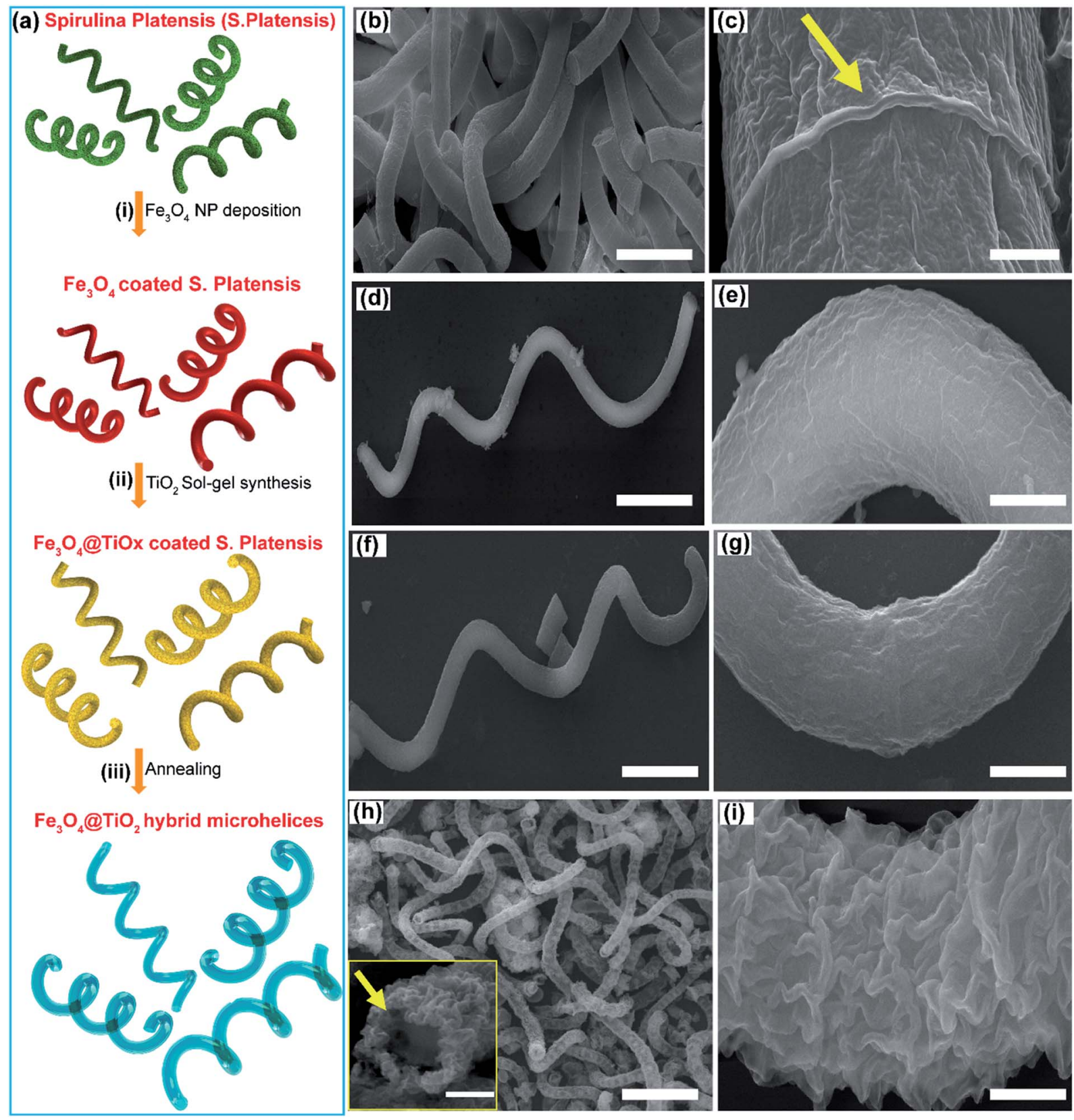

Fig. 1 Fabrication of hybrid $\mathrm{Fe}_{3} \mathrm{O}_{4} \mathrm{aTiO}_{2}$ microhelices. (a) Fabrication scheme to develop hybrid microrobots. (b) SEM image of many overlapping bio-templates and (c) the magnified image showing the presence of a rough morphology (depicted with arrow). (d and e) SEM images showing coated $\mathrm{Fe}_{3} \mathrm{O}_{4}$ bio-templates and ( $\mathrm{f}$ ) and (g) showing SEM images of $\mathrm{Fe}_{3} \mathrm{O}_{4} \mathrm{aTiO} \mathrm{T}_{x}$ coated bio-templates. (h) SEM image showing many overlapping hybrid $\mathrm{Fe}_{3} \mathrm{O}_{4} \mathrm{CTiO}_{2}$ microhelices obtained after annealing. The inset shows the formation of hollow microhelices after annealing. (i) A magnified SEM image showing the presence of rough and irregular morphology of hybrid $\mathrm{Fe}_{3} \mathrm{O}_{4} @ \mathrm{TiO}_{2}$ microhelices. Scale bars: (b) $20 \mu \mathrm{m}$, (c) 1 $\mu \mathrm{m}$, (d) $30 \mu \mathrm{m}$, (e) $3 \mu \mathrm{m}$, (f) $30 \mu \mathrm{m}$, (g) $4 \mu \mathrm{m}$, (h) $20 \mu \mathrm{m}$, inset $1 \mu \mathrm{m}$ and (i) $1 \mu \mathrm{m}$ micropillars and (d) hybrid microhelices; (e) SEM image produced by performing a FIB cut on a hybrid micropillar. Scale bars indicate $10 \mu \mathrm{m}(\mathrm{a}), 2 \mu \mathrm{m}(\mathrm{b}-\mathrm{d})$.

S5 †) showed a good crystallinity and all the peaks were assigned to either $\mathrm{Fe}_{3} \mathrm{O}_{4}$ or $\mathrm{TiO}_{2}$. The diffraction peaks obtained at $30.3^{\circ}$, $35.9^{\circ}, 37.2^{\circ}, 43.6^{\circ}, 53.6^{\circ}, 57.6^{\circ}, 62.9^{\circ}$ and $75.3^{\circ}$ can be assigned to the (220), (311), (222), (400), (511), (440) and (533) crystal planes of cubic $\mathrm{Fe}_{3} \mathrm{O}_{4}$ phase (JCPDS no. 75-0449). ${ }^{39}$ Similarly, peaks at $25.4^{\circ}, 38.1^{\circ}, 38.7^{\circ}, 48.1^{\circ}, 54.2^{\circ}, 55.1^{\circ}, 69.1^{\circ}, 70.3^{\circ}$ and $82.3^{\circ}$ can be assigned to the (101), (004), (112), (200), (105),
(211), (116), (220) and (312) crystal planes of the anatase $\mathrm{TiO}_{2}$ phase (JCPDS no. 73-1764). ${ }^{20}$

Fig. 2b shows a high angle annular dark field (HAADF) scanning transmission electron microscopy (STEM) image of a single hybrid microhelix. From this image we can observe a uniform tubular helix with a rough morphology. The inset shows its corresponding selected area electron diffraction (SAED) pattern. High-resolution transmission electron 

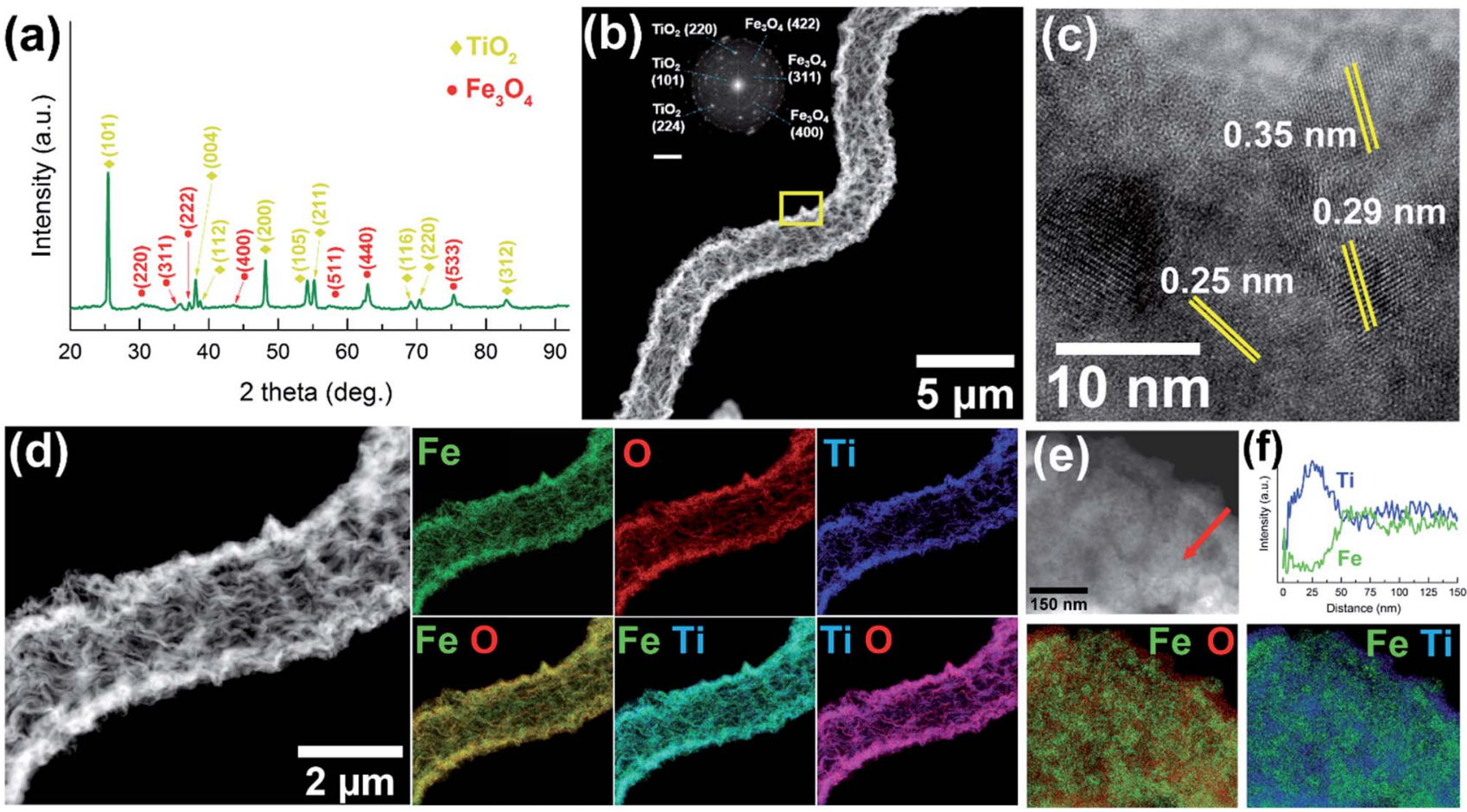

Fig. 2 Structural characterisation of hybrid $\mathrm{Fe}_{3} \mathrm{O}_{4} \mathrm{CTiO}_{2}$ microhelices. (a) XRD patterns obtained for hybrid $\mathrm{Fe}_{3} \mathrm{O}_{4} \mathrm{aTiO}_{2}$ microhelices. (b) $\mathrm{HAADF}$ STEM image obtained for a hybrid microhelix and the inset shows its corresponding SAED pattern. (c) HRTEM image obtained in the area marked in (b), showing the presence of cubic $\mathrm{Fe}_{3} \mathrm{O}_{4}$ and anatase $\mathrm{TiO}_{2}$. (d) HAADF STEM image obtained for a single hybrid helix and the corresponding EDX maps obtained for $\mathrm{Fe}, \mathrm{O}$ and $\mathrm{Ti}$, with the superimposed images clearly showing a uniform distribution of all elements on its surface. (e) HRTEM image obtained at the edge of a hybrid microhelix and its corresponding EDX maps showing the presence of a shell composed of Ti and $\mathrm{O}$. (f) Plot showing the distribution of Ti and Fe elements along the region marked in (e), which clearly depicts the presence of a $50 \mathrm{~nm}$ thick edge that is rich in $\mathrm{Ti}$.

microscopy (HRTEM) analysis was performed on the area marked in Fig. $2 \mathrm{~b}$ and is presented in Fig. $2 \mathrm{c}$. From this, we can observe the $d$-spacing of $0.250 \mathrm{~nm}$ and $0.293 \mathrm{~nm}$, which corresponds to the cubic (311) and (220) $\mathrm{Fe}_{3} \mathrm{O}_{4}$ planes, and the $d$ value of $0.350 \mathrm{~nm}$, which corresponds to the anatase (101) $\mathrm{TiO}_{2}$ plane. A HAADF STEM image of a hybrid $\mathrm{Fe}_{3} \mathrm{O}_{4} @ \mathrm{TiO}_{2}$ microhelix is presented in Fig. 2d, showing a tubular structure with a wall thickness of around $300 \mathrm{~nm}$. From the corresponding energy-dispersive X-ray analysis (EDX) mappings, we can observe a uniform distribution of all elements over the microhelices. The corresponding EDX spectrum obtained from this region is provided in Fig. S6. $\dagger$ Additionally, a magnified HRTEM image of the hybrid microhelix was obtained near the core-shell region and is presented in Fig. 2e. Fig. 2f shows the intensity of Fe and Ti signals obtained along the region marked in Fig. 2e. From this plot we can observe that the outermost edge (about $50 \mathrm{~nm}$ thick) of the hybrid structure consists predominantly of $\mathrm{Ti}$, with an increasing amount of Fe signal towards the core. These results are further corroborated by the superimposed EDX mappings obtained for Fig. 2e, from where we can clearly observe that the hybrid structure consists of a shell composed of $\mathrm{Ti}$ and $\mathrm{O}$. From these characterization results, we can conclude that our hybrid microhelices are tubular structures with a rough morphology, and are composed of a $50 \mathrm{~nm}$ thick $\mathrm{TiO}_{2}$ shell and a hollow $\mathrm{Fe}_{3} \mathrm{O}_{4}$ core with a wall thickness of about $300 \mathrm{~nm}$. In this work, we also fabricated microhelices composed of only $\mathrm{Fe}_{3} \mathrm{O}_{4}$ or $\mathrm{TiO}_{2}$ and compared their optical and photocatalytic properties to the core-shell $\mathrm{Fe}_{3} \mathrm{O}_{4} @ \mathrm{TiO}_{2}$ hybrid microstructures.

The light absorption properties of microhelices composed of $\mathrm{Fe}_{3} \mathrm{O}_{4}, \mathrm{TiO}_{2}$ and $\mathrm{Fe}_{3} \mathrm{O}_{4} @ \mathrm{TiO}_{2}$ were investigated using a UVvisible diffuse reflectance spectra (DRS). From Fig. 3a, we can observe that for $\mathrm{TiO}_{2}$ microstructures, light absorption is limited to the UV-light region, whereas $\mathrm{Fe}_{3} \mathrm{O}_{4}$ microstructures exhibit absorption in the UV and visible light region. The hybrid microstructures display a strong absorbance in the UV and visible light region, similar to the $\mathrm{Fe}_{3} \mathrm{O}_{4}$ microstructures. The bandgaps of these microhelices were calculated using the Kubelka-Munk function $\left((\alpha h \nu)^{1 / 2} v s\right.$. photon energy $\left.(h \nu)\right)$ for the indirect bandgap semiconductors (Fig. $3 \mathrm{~b}$ ). The bandgaps were estimated to be $1.6 \mathrm{eV}, 3.2 \mathrm{eV}$ and $2.2 \mathrm{eV}$ for $\mathrm{Fe}_{3} \mathrm{O}_{4}, \mathrm{TiO}_{2}$ and $\mathrm{Fe}_{3} \mathrm{O}_{4} @ \mathrm{TiO}_{2}$ microstructures respectively, which are consistent with existing literature. ${ }^{41,43,44}$ From the bandgap value of $2.2 \mathrm{eV}$, we can conclude that $\mathrm{Fe}_{3} \mathrm{O}_{4} @ \mathrm{TiO}_{2}$ microstructures can be used as photocatalysts under UV and visible light.

We then investigated the ability of our microhelices to degrade an organic pollutant, rhodamine B (RhB), using UVvisible light and natural sunlight. From Fig. $3 \mathrm{c}$ we can observe that all three types of microhelices, i.e. $\mathrm{Fe}_{3} \mathrm{O}_{4}, \mathrm{TiO}_{2}$, and the hybrid $\mathrm{Fe}_{3} \mathrm{O}_{4} @ \mathrm{TiO}_{2}$ were able to gradually degrade the $\mathrm{RhB}$ dye 
(a)

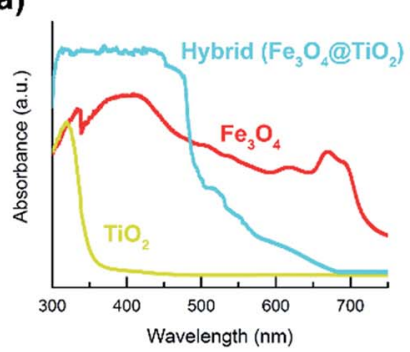

(d)

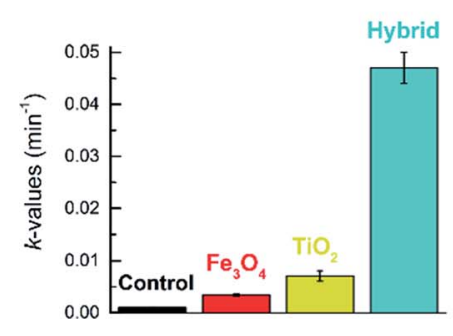

(b)

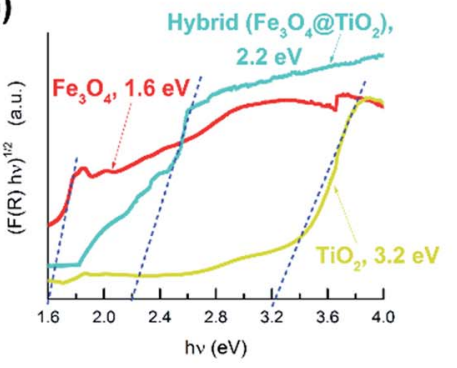

(e)

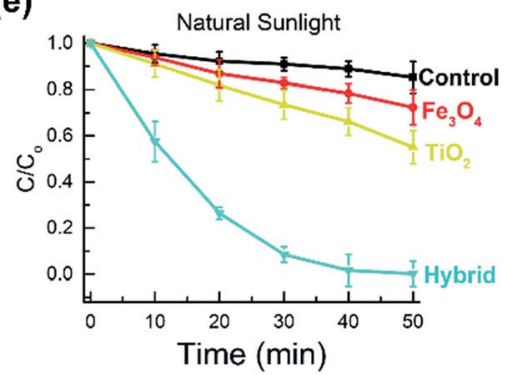

(c)

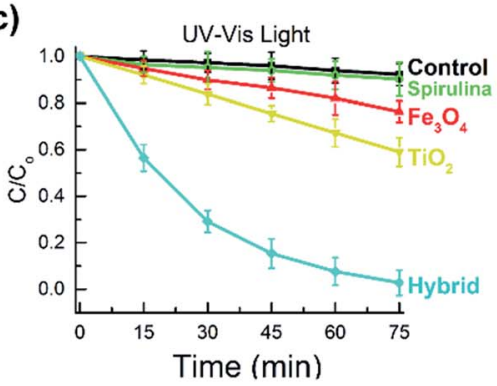

(f)

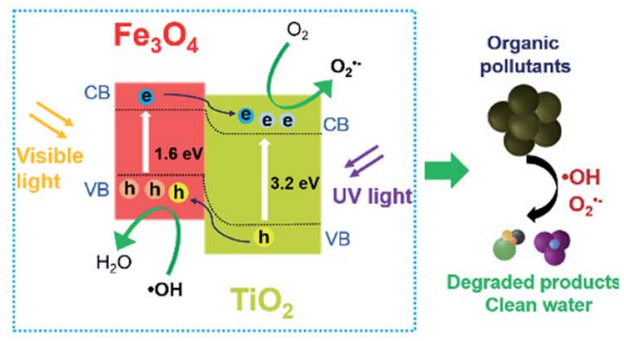

Fig. 3 Photocatalytic characterisation of hybrid $\mathrm{Fe}_{3} \mathrm{O}_{4} \mathrm{CTiO}_{2}$ microhelices. (a) UV-Vis DRS spectra obtained for $\mathrm{Fe}_{3} \mathrm{O}_{4}$, $\mathrm{TiO}_{2}$ and core-shell $\mathrm{Fe}_{3} \mathrm{O}_{4} \mathrm{OTiO}_{2}$ microhelices and (b) the corresponding Kubelka-Munk plot. BFO NS and NW samples XRD patterns obtained for hybrid Fe ${ }^{-}$ $\mathrm{O}_{4} \mathrm{OTiO}_{2}$ microhelices. (c) Photocatalytic degradation curves of RhB obtained under UV-visible light and mechanical stirring. (d) Comparison of degradation rate constants of RhB obtained under UV-visible light and mechanical stirring. (e) Photocatalytic degradation curves of RhB obtained under natural sunlight and mechanical stirring. (f) Scheme showing the mechanism behind the photocatalytic degradation of organic pollutants under UV-visible light using hybrid $\mathrm{Fe}_{3} \mathrm{O}_{4} \mathrm{aTiO}_{2}$ microstructures.

under UV-visible light over 75 minutes. In contrast, the control sample (without any microhelices) and the sample with only the bio-template of $S$. platensis displayed a negligible response. The hybrid microstructures exhibited a $97 \%$ RhB degradation rate, in comparison to a $41 \%$ degradation rate obtained for $\mathrm{TiO}_{2}$ microstructures and $24 \%$ for $\mathrm{Fe}_{3} \mathrm{O}_{4}$ microstructures. A quantitative analysis of these degradation rates is given by comparing their reaction rate constant $k$, which can be defined by eqn (1):

$$
\ln \frac{C_{\mathrm{o}}}{C}=k t
$$

where $C_{\mathrm{o}}$ is the initial $\mathrm{RhB}$ concentration, and $C$ is the $\mathrm{RhB}$ concentration at time $t$. This calculation is based on the assumption that the kinetics of the RhB degradation reaction catalyzed by the microstructures are (pseudo)-first-order reactions. ${ }^{20,26}$ These results are presented in Fig. $3 \mathrm{~d}$, where we can observe that the $\mathrm{Fe}_{3} \mathrm{O}_{4}$ microstructures displayed the slowest photocatalytic rate constant of $0.00345 \mathrm{~min}^{-1}$. In comparison, $\mathrm{TiO}_{2}$ microstructures with a $k$-value of $0.00707 \mathrm{~min}^{-1}$, were almost twice as fast in degrading $\mathrm{RhB}$. The photocatalytic rate constant obtained for hybrid $\mathrm{Fe}_{3} \mathrm{O}_{4} @ \mathrm{TiO}_{2}$ microstructures $\left(0.04705 \mathrm{~min}^{-1}\right)$ outperformed $\mathrm{TiO}_{2}$ and $\mathrm{Fe}_{3} \mathrm{O}_{4}$ microstructures by 6.7 and 13.6 times respectively. Similar results were obtained when the photocatalytic reactions were conducted under natural sunlight, where the hybrid structures degraded $99.8 \%$ of $\mathrm{RhB}$ in 50 minutes (Fig. 3e). In Table S1† we have compared our enhanced results with other reported magnetic photocatalysts.

To explain the superior performance of the hybrid $\mathrm{Fe}_{3} \mathrm{O}_{4} @$ @$\mathrm{TiO}_{2}$ microstructures to the $\mathrm{Fe}_{3} \mathrm{O}_{4}$ or $\mathrm{TiO}_{2}$ microstructures, we first explored the underlying mechanism behind photocatalysis. When a semi-conductor is irradiated with light, electrons are excited from the valence band (VB) to its conduction band (CB), leaving behind holes in its VB and creating electron-hole pairs on its surface. These electron-hole pairs initiate a series of redox reactions by converting oxygen and water into highly reactive oxygen species, such as superoxide $\left(\mathrm{O}_{2}{ }^{-}\right)$and hydroxyl $\left({ }^{\circ} \mathrm{OH}\right)$ radicals. These radicals are directly responsible for photocatalytic degradation of organic molecules into harmless products (Fig. 3f). With a bandgap of $1.6 \mathrm{eV}, \mathrm{Fe}_{3} \mathrm{O}_{4}$ microstructures are capable of absorbing UV and visible light to create electron-hole pairs. However, this small bandgap leads to an increased electron-hole recombination rate, which significantly reduces their photocatalytic efficiency. In contrast, $\mathrm{TiO}_{2}$ microstructures are wide bandgap photocatalysts $(3.2 \mathrm{eV})$ with a light absorption limited to the UV region, which explains their poor photocatalytic performance. Combining small and wide bandgap photocatalysts improves the photocatalytic efficiency by absorbing a wide spectrum of light, and by reducing the recombination rate of photogenerated electron-hole pairs. In our study, the hybrid microstructures' enhanced photocatalytic performance can be explained by their strong absorption of UV and visible light, as well as the fast interfacial charge transfer between $\mathrm{Fe}_{3} \mathrm{O}_{4}$ and $\mathrm{TiO}_{2}$. Under UV-visible light or natural sunlight, photogenerated electrons in $\mathrm{Fe}_{3} \mathrm{O}_{4}$ are excited from its VB to its $\mathrm{CB}$, and by absorbing UV light, the electrons in $\mathrm{TiO}_{2}$ are excited to its CB. When $\mathrm{Fe}_{3} \mathrm{O}_{4}$ and $\mathrm{TiO}_{2}$ are brought in contact with each other, the excited electrons in the $\mathrm{CB}$ of $\mathrm{Fe}_{3} \mathrm{O}_{4}$ are transferred to the $\mathrm{CB}$ of $\mathrm{TiO}_{2}$, which is an electron acceptor that readily accepts these electrons. Similarly, the holes left 
(a)

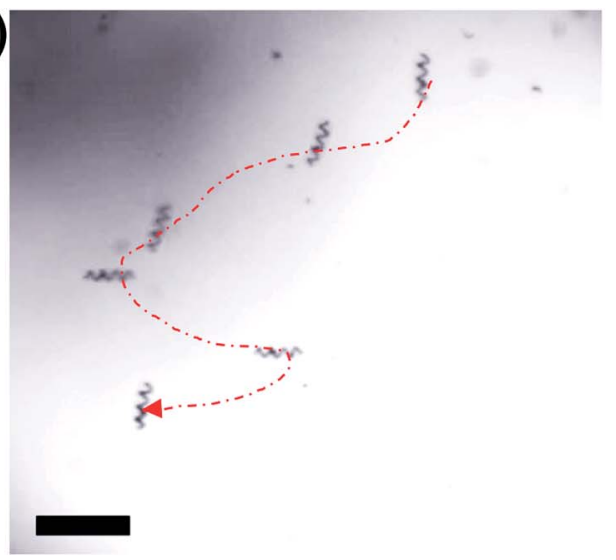

(C) Influence of Swimming Speed on RhB degradation under UV-Vis Light

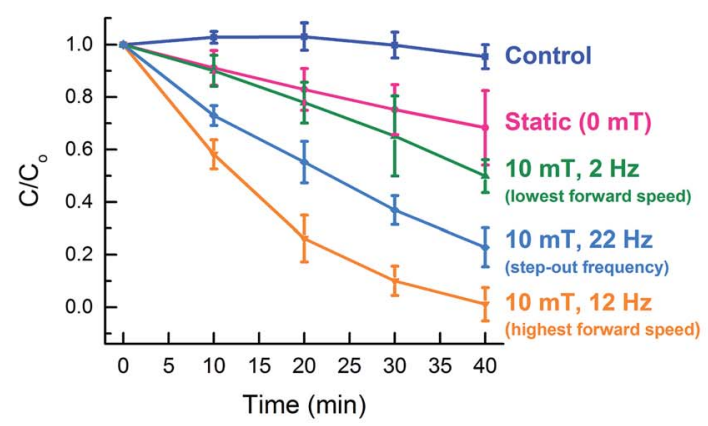

(e)

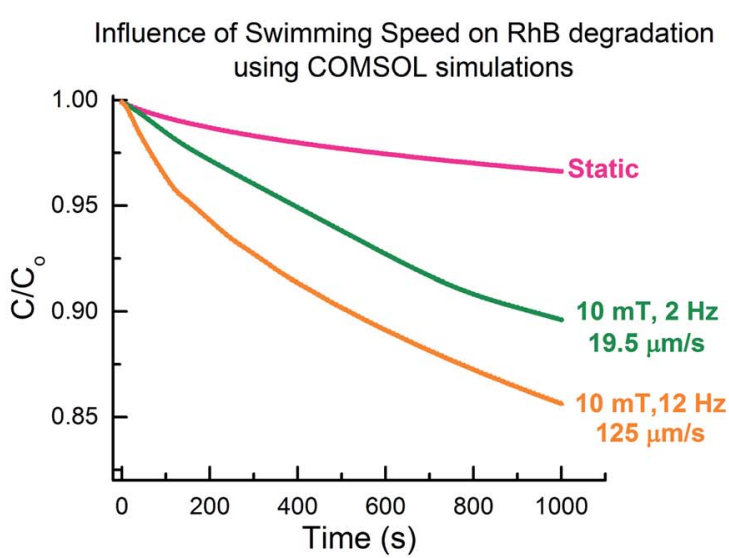

(b)

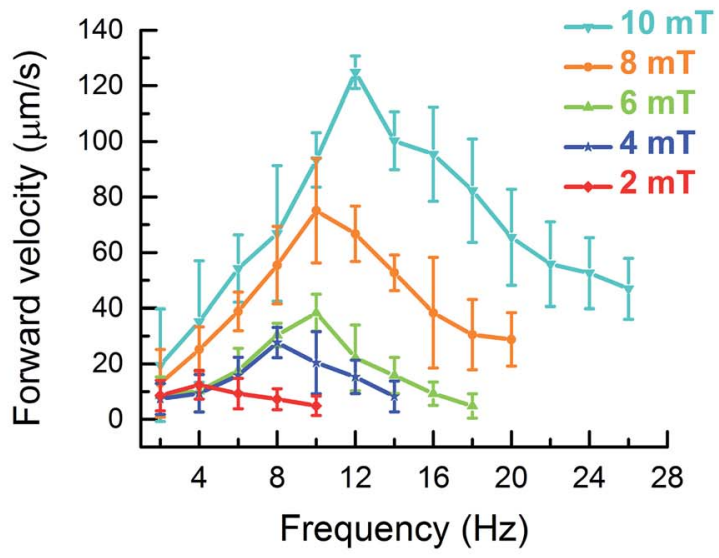

(d)

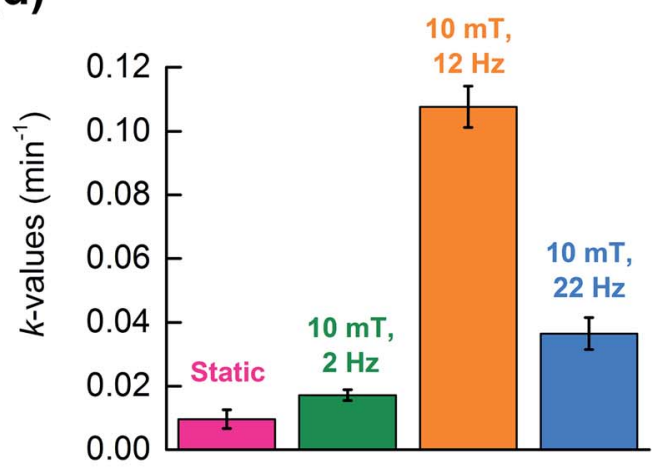

(f)

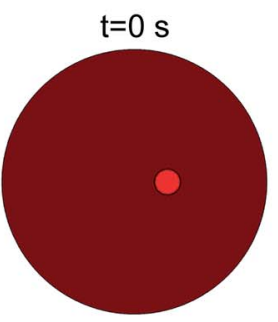

$19.5 \mu \mathrm{m} / \mathrm{s}, \mathrm{t}=1000 \mathrm{~s}$

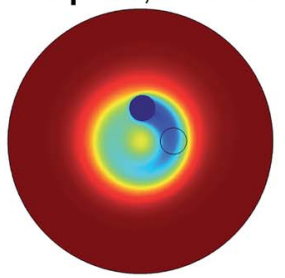

Static, $\mathrm{t}=1000 \mathrm{~s}$

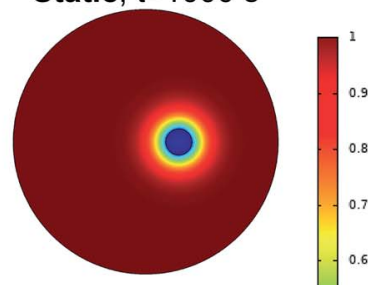

$125 \mu \mathrm{m} / \mathrm{s}, \mathrm{t}=1000 \mathrm{~s}$

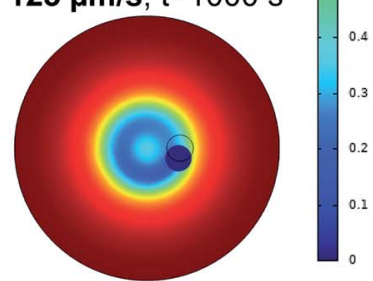

Fig. 4 Propulsion of hybrid $\mathrm{Fe}_{3} \mathrm{O}_{4} \mathrm{aTiO}_{2}$ microhelices under magnetic fields and the corresponding photocatalytic degradation. (a) Time-lapse image showing magnetic manipulation and steering of a hybrid microhelix (10 mT and $12 \mathrm{~Hz})$, scale bar $50 \mu \mathrm{m}$. (b) Swimming speeds obtained for hybrid microhelices under different magnetic field strengths and rotational frequencies $(n=6)$. (c) Photocatalytic degradation obtained for hybrid microhelices using UV-visible light and under continuous magnetic field induced swimming at different speeds and (d) the corresponding photocatalytic degradation rate constants $(n=5$ ). (e) Simulated degradation curves obtained under different swimming speeds. (f) COMSOL simulation results showing the organic pollutant degradation efficiencies obtained under different swimming speeds. The scale bar represents the concentration of organic pollutant, $\mathrm{RhB}$, as $C / C_{0}$, where the dark blue color represents complete RhB degradation.

behind in the VB of $\mathrm{TiO}_{2}$ are transferred to the $\mathrm{VB}$ of $\mathrm{Fe}_{3} \mathrm{O}_{4}$, leading to a separation of photogenerated electron-hole pairs and a suppression of their recombination. ${ }^{\mathbf{1 8 , 2 4}}$ Hence, in this narrow-wide bandgap photocatalysis system, the photogenerated electron-hole pairs have an increased lifetime of the charge carriers, resulting in their improved performance over 
a narrow or wide bandgap photocatalyst. We also studied the reusability performance of our photocatalytic microstructures over several consecutive cleaning cycles. These results are presented in Fig. S7, $\dagger$ where it can be observed that the hybrid microstructures exhibited a good stability and reusability property over multiple runs.

To develop cost-effective photocatalysts for practical applications, it is important that they can be easily collected for reuse to avoid secondary pollution due to their loss. The use of $\mathrm{Fe}_{3} \mathrm{O}_{4}$ NPs gives our helical microstructures superparamagnetic properties which can be used for their magnetic control (Fig. S8 $\dagger$ ). To achieve this, we used wireless magnetic fields to propel our hybrid $\mathrm{Fe}_{3} \mathrm{O}_{4} @ \mathrm{TiO}_{2}$ microstructures. During our study we observed that the hybrid microstructures could be wirelessly actuated and displayed a corkscrew motion. Fig. 4a, provides a time-lapse image showing a single hybrid $\mathrm{Fe}_{3} \mathrm{O}_{4} @$ $\mathrm{TiO}_{2}$ microstructure performing a controlled corkscrew motion on a pre-defined trajectory under rotating magnetic fields (10 $\mathrm{mT}$ and $12 \mathrm{~Hz}$ ). We were also able to control and steer a swarm of several hybrid microstructures to specified locations (Fig. S9, Videos S1 and S2 $\dagger$ ). We then studied the swimming characteristics of hybrid microhelices as a function of different rotating magnetic fields and frequencies (Fig. $4 \mathrm{~b}$ and Videos S3-S5†). From these plots we can observe that the hybrid helices exhibited increasing forward velocities with increasing magnetic field strength before reaching their step-out frequency. The fastest swimming speed was obtained at a magnetic field strength of $10 \mathrm{mT}$ with a rotational frequency of $12 \mathrm{~Hz}$, and the slowest velocity was obtained at $2 \mathrm{~Hz}$ with the step-out frequency set-in at $14 \mathrm{~Hz}$.

Next, we investigated the influence exerted by the swimming characteristics of the microstructures on their photocatalytic degradation efficiency. We designed special chambers to conduct photocatalytic experiments on a swarm of hybrid microstructures using a UV-visible light source which we integrated into our magnetic manipulation system. We designed this experiment to study the photocatalytic efficiency of $\mathrm{Fe}_{3}$ $\mathrm{O}_{4} @ \mathrm{TiO}_{2}$ microstructures under static conditions (no magnetic fields), at their lowest $\left(20 \mu \mathrm{m} \mathrm{s}^{-1}\right.$ at $\left.10 \mathrm{mT}, 2 \mathrm{~Hz}\right)$ and highest $\left(125 \mu \mathrm{m} \mathrm{s}^{-1}\right.$ at $\left.10 \mathrm{mT}, 12 \mathrm{~Hz}\right)$ forward velocities, and in the stepout frequency region, where they demonstrated intermediary speeds $\left(56 \mu \mathrm{m} \mathrm{s}^{-1}\right.$ at $10 \mathrm{mT}, 22 \mathrm{~Hz}$ ). Fig. $4 \mathrm{c}$ presents the results obtained during this study, where we can clearly observe that the photocatalytic degradation efficiency is highly dependent on the swimming characteristics exhibited by the hybrid microstructures. Static samples displayed the worst photocatalytic performance, probably due to agglomeration and poor diffusion. Hybrid helices swimming at low and intermediary speeds exhibited an improved photocatalytic performance in comparison to the static samples. Additionally, the hybrid microstructures swimming at the highest speed exhibited the most enhanced photocatalytic performance ( $k$-value of $0.107 \mathrm{~min}^{-1}$ ). The reaction rate constants of these results can be found in Fig. 3d. From this plot we can clearly observe that the microstructures swimming at the highest speed $(10 \mathrm{mT}, 12 \mathrm{~Hz})$ displayed a photocatalytic degradation rate 2.9 times higher than the intermediary speed and about 6.3 times higher than the slowest swimming microhelices. It is also important to note that in comparison to static microhelices, mobile microhelices swimming at the highest speed displayed an increase in the $\mathrm{RhB}$ degradation rate of up 11.2 times. These results for mobile swimmers can be explained by an enhanced diffusive mass transport and the subsequent rapid intermixing of the photogenerated radicals with the surrounding RhB molecules, which leads to their superior catalytic efficiency. ${ }^{34,45}$ This further explains the increasing catalytic efficiency obtained under increasing swimming speeds. We further confirmed the good re-usability performance of our hybrid samples under continuous magnetic propulsion (Fig. S10†).

To verify our hypothesis we used COMSOL Multiphysics software to simulate the influence of microswimmers' motion on their subsequent catalytic reaction performance (Fig. 4e). From this plot we can observe that the most enhanced cleaning rate was obtained for swimmers swimming at the highest speed $\left(125 \mu \mathrm{m} \mathrm{s}^{-1}\right)$. Fig. $4 \mathrm{f}$ and $\mathrm{S} 11 \dagger$ present a collage of time-lapse images taken from the COMSOL simulations that qualitatively show the organic pollutant degradation performance obtained for different swimming speeds after 1000 seconds. Here, the outer circle represents a closed container filled with organic pollutant and the small circle near the centre represents a swarm of microswimmers swimming on a circular path. These microswimmers are assumed to have a constant surface concentration of radicals, which leads to the degradation of the organic pollutant. The color scale represents the relative organic pollutant concentration as $C / C_{\mathrm{o}}$, where $C_{\mathrm{o}}$ is the initial pollutant concentration of $4 \mathrm{mg} \mathrm{L}^{-1}$. These results indicate that the swimmers swimming at $125 \mu \mathrm{m} \mathrm{s}^{-1}$ demonstrate the most enhanced degradation rate due to the larger volume of pollutants coming into direct contact with the swimmers. For the static microswimmers, the degradation rate is diffusion limited and therefore much smaller. These results are also presented in Videos S6-S8, $\dagger$ and highlight the benefits of continuously actuating the microswimmers at their optimal speed in order to obtain the most enhanced water remediation performance.

\section{Conclusion}

In this work, we have used bio-templating and sol-gel synthesis to fabricate core-shell $\mathrm{Fe}_{3} \mathrm{O}_{4} @ \mathrm{TiO}_{2}$ microhelices for UV-visible light driven photocatalytic water remediation. These hybrid microhelices developed in this work have a bandgap of $2.2 \mathrm{eV}$ and were able to absorb UV and visible light, and degrade over $97 \%$ of RhB dye within 75 minutes ( $k$-value of $0.047 \mathrm{~min}^{-1}$ ). We also demonstrated their use under natural sunlight to photocatalytically degrade $99.8 \%$ of $\mathrm{RhB}$ in 50 minutes. Magnetic characterization revealed a paramagnetic nature of the hybrid microhelices, and wireless rotating magnetic fields were employed to precisely propel and steer them in a corkscrew motion. We also investigated the effect of continuous magnetic propulsion on the photocatalytic reaction rates and discovered that mobile swimmers exhibited superior performance over the static microhelices. The microstructures swimming at the highest speed of $125 \mu \mathrm{m} \mathrm{s}^{-1}$ exhibited 11.2 times higher reaction rates than the static microhelices $\left(k\right.$-value of $0.107 \mathrm{~min}^{-1}$ 
vs. $0.009 \mathrm{~min}^{-1}$ respectively). In addition, the microhelices swimming at intermediary speeds of $56 \mu \mathrm{m} \mathrm{s}^{-1}$ and the lowest speeds of $20 \mu \mathrm{m} \mathrm{s}^{-1}$ demonstrated 6.3 times and 2.9 times lower photocatalytic efficiency than the fastest swimmers. The superior performance of mobile swimmers over the static microhelices can be explained by their enhanced diffusive mass transport and rapid intermixing. We also corroborated our experimental findings by performing COMSOL simulations to study the influence of the swimming speed of a swarm of microswimmers on the catalytic degradation rate of an organic pollutant.

\section{Conflicts of interest}

There are no conflicts to declare.

\section{Acknowledgements}

This work was financed by the European Research Council Starting Grant Magnetoelectric Chemonanorobotics for Chemical and Biomedical Applications (ELECTROCHEMBOTS), by the ERC grant agreement no. 336456. X. W. acknowledges financial support from China Scholarship Council (No. 201504910817). The authors would like to acknowledge the Scientific Center for Optical and Electron Microscopy (ScopeM), the Institute of Geochemistry and Petrology, and the FIRST laboratory, ETH Zurich for their technical support. The authors would like to acknowledge Pascal Gschwend from Particle Technology Lab of ETH Zurich for help with DRS measurements.

\section{Notes and references}

1 A. Matilainen, M. Vepsäläinen and M. Sillanpää, Adv. Colloid Interface Sci., 2010, 159, 189.

2 K. Ikehata, N. Jodeiri Naghashkar and M. Gamal El-Din, Ozone: Sci. Eng., 2006, 28, 353.

3 L. Kovalova, H. Siegrist, U. von Gunten, J. Eugster, M. Hagenbuch, A. Wittmer, R. Moser and C. S. McArdell, Environ. Sci. Technol., 2013, 47, 7899.

4 A. Joss, E. Keller, A. C. Alder, A. Göbel, C. S. McArdell, T. Ternes and H. Siegrist, Water Res., 2005, 39, 3139.

5 R. O. A. d. Lima, A. P. Bazo, D. M. F. Salvadori, C. M. Rech, D. d. P. Oliveira and d. A. G. Umbuzeiro, Mutat. Res., Genet. Toxicol. Environ. Mutagen., 2007, 626, 53.

6 K.-T. Chung, Mutat. Res., Genet. Toxicol. Environ. Mutagen., 1983, 114, 269.

7 M. E. Fracasso, R. Leone, F. Brunello, C. Monastra, F. Tezza and P. V. Storti, Mutat. Res., Genet. Toxicol., 1992, 298, 91.

8 J. Margot, C. Kienle, A. Magnet, M. Weil, L. Rossi, L. F. de Alencastro, C. Abegglen, D. Thonney, N. Chèvre, M. Schärer and D. A. Barry, Sci. Total Environ., 2013, 461-462, 480.

9 R. I. L. Eggen, J. Hollender, A. Joss, M. Schärer and C. Stamm, Environ. Sci. Technol., 2014, 48, 7683.

10 D. Sudha and P. Sivakumar, Chem. Eng. Process., 2015, 97, 112.
11 D. B. Miklos, C. Remy, M. Jekel, K. G. Linden, J. E. Drewes and U. Hübner, Water Res., 2018, 139, 118.

12 S.-J. Xia, F.-X. Liu, Z.-M. Ni, J.-L. Xue and P.-P. Qian, J. Colloid Interface Sci., 2013, 405, 195.

13 M. N. Chong, B. Jin, C. W. K. Chow and C. Saint, Water Res., 2010, 44, 2997.

14 R. Thiruvenkatachari, S. Vigneswaran and I. S. Moon, Korean J. Chem. Eng., 2008, 25, 64.

15 Y. Meng, X. Zhang, G. Pan, S. Xia and Z. Ni, J. Hazard. Mater., 2018, 350, 144.

16 K. Nakata and A. Fujishima, J. Photochem. Photobiol., C, 2012, 13, 169.

17 J. Schneider, M. Matsuoka, M. Takeuchi, J. Zhang, Y. Horiuchi, M. Anpo and D. W. Bahnemann, Chem. Rev., 2014, 114, 9919.

18 Y. L. Pang, S. Lim, H. C. Ong and W. T. Chong, Appl. Catal., A, 2014, 481, 127.

19 F. Mushtaq, M. Guerrero, M. S. Sakar, M. Hoop, A. M. Lindo, J. Sort, X. Chen, B. J. Nelson, E. Pellicer and S. Pane, J. Mater. Chem. A, 2015, 3, 23670.

20 F. Mushtaq, A. Asani, M. Hoop, X.-Z. Chen, D. Ahmed, B. J. Nelson and S. Pané, Adv. Funct. Mater., 2016, 26, 6995.

21 J. Low, J. Yu, M. Jaroniec, S. Wageh and A. A. Al-Ghamdi, Adv. Mater., 2017, 29, 1601694.

22 M. R. D. Khaki, M. S. Shafeeyan, A. A. A. Raman and W. M. A. W. Daud, J. Environ. Manage., 2017, 198, 78.

23 F. Sordello, I. Berruti, C. Gionco, M. C. Paganini, P. Calza and C. Minero, Appl. Catal., B, 2019, 245, 159.

24 S. G. Kumar and L. G. Devi, J. Phys. Chem. A, 2011, 115, 13211.

25 Y. Ma, X. Wang, Y. Jia, X. Chen, H. Han and C. Li, Chem. Rev., 2014, 114, 9987.

26 F. Mushtaq, X. Chen, M. Hoop, H. Torlakcik, E. Pellicer, J. Sort, C. Gattinoni, B. J. Nelson and S. Pané, iScience, 2018, 4, 236.

27 H. Cheng, J. Wang, Y. Zhao and X. Han, RSC Adv., 2014, 4, 47031.

28 B. Mazinani, A. K. Masrom, A. Beitollahi and R. Luque, Ceram. Int., 2014, 40, 11525.

29 B. Seo, C. Lee, D. Yoo, P. Kofinas and Y. Piao, $R S C A d v ., 2017$, 7, 9587.

30 F. Mushtaq, X. Chen, H. Torlakcik, C. Steuer, M. Hoop, E. C. Siringil, X. Marti, G. Limburg, P. Stipp, B. J. Nelson and S. Pané, Adv. Mater., 2019, 31, 1901378.

31 F. Mushtaq, H. Torlakcik, M. Hoop, B. Jang, F. Carlson, T. Grunow, N. Läubli, A. Ferreira, X.-Z. Chen, B. J. Nelson and S. Pané, Adv. Funct. Mater., 2019, 29, 1808135.

32 P. O. Gendron, F. Avaltroni and K. J. Wilkinson, J. Fluoresc., 2008, 18, 1093.

33 K. E. Peyer, L. Zhang and B. J. Nelson, Nanoscale, 2013, 5, 1259.

34 Y. Zhang, K. Yan, F. Ji and L. Zhang, Adv. Funct. Mater., 2018, 28, 1806340.

35 L. Zhang, J. J. Abbott, L. Dong, B. E. Kratochvil, D. Bell and B. J. Nelson, Appl. Phys. Lett., 2009, 94, 064107. 
36 X. Yan, Q. Zhou, M. Vincent, Y. Deng, J. Yu, J. Xu, T. Xu, T. Tang, L. Bian, Y.-X. J. Wang, K. Kostarelos and L. Zhang, Sci. Robot., 2017, 2, eaaq1155.

37 T. Zhang, W. Wang, D. Zhang, X. Zhang, Y. Ma, Y. Zhou and L. Qi, Adv. Funct. Mater., 2010, 20, 1152.

38 S. Sotiropoulou, Y. Sierra-Sastre, S. S. Mark and C. A. Batt, Chem. Mater., 2008, 20, 821.

39 Y. D. Tu, Z. Zhou, R. J. Yan, Y. P. Gan, W. Z. Huang, X. X. Weng, H. Huang, W. K. Zhang and X. Y. Tao, RSC Adv., 2012, 2, 10585.

40 K. Kamata, Z. Piao, S. Suzuki, T. Fujimori, W. Tajiri, K. Nagai, T. Iyoda, A. Yamada, T. Hayakawa, M. Ishiwara, S. Horaguchi, A. Belay, T. Tanaka, K. Takano and M. Hangyo, Sci. Rep., 2014, 4, 4919.
41 J. Liu, S. Yang, W. Wu, Q. Tian, S. Cui, Z. Dai, F. Ren, X. Xiao and C. Jiang, ACS Sustainable Chem. Eng., 2015, 3, 2975.

42 X. Cao, Y. Chen, S. Jiao, Z. Fang, M. Xu, X. Liu, L. Li, G. Pang and S. Feng, Nanoscale, 2014, 6, 12366.

43 S. Rajamohan, V. Kumaravel, R. Muthuramalingam, S. Ayyadurai, A. Abdel-Wahab, B. Sub Kwak, M. Kang and S. Sreekantan, New J. Chem., 2017, 41, 11722.

44 P. Kumar, C. Joshi, A. Barras, B. Sieber, A. Addad, L. Boussekey, S. Szunerits, R. Boukherroub and S. L. Jain, Appl. Catal., B, 2017, 205, 654.

45 J. Yu, B. Wang, X. Du, Q. Wang and L. Zhang, Nat. Commun., 2018, 9, 3260. 